Gut. 1980, 21, 851-854

\title{
Bacteriological and endotoxin studies in cases of ulcerative colitis submitted to surgery
}

\author{
K R PALMER, B I DUERDEN, AND C D HOLDSWORTH \\ From the Department of Medical Microbiology and Gastroenterology Unit, Royal Hallamshire Hospital, \\ Sheffield
}

SUMMARY Culture for bacteria and assays for endotoxin were performed on specimens of mesenteric and peripheral venous blood from eight patients with ulcerative colitis who underwent surgery for severe uncontrolled disease. No significant bacteraemia occurred in either portal or systemic blood. Systemic endotoxaemia developed in three patients during surgery but occurred before bowel mobilisation only in the one patient whose colitis was complicated by hyposplenism. Mesenteric endotoxaemia occurred in only three patients before bowel mobilisation, but was detected during surgery in two of the three patients who developed systemic endotoxaemia. We conclude that, contrary to earlier reports, portal bacteraemia must be infrequent in ulcerative colitis. Systemic endotoxaemia does, however, occur in a significant proportion of cases during colectomy. Although in the patients studied this led to no clinical problems, it is likely to have been the precipitating factor for the syndrome of disseminated intravascular coagulation (DIC) that we have previously observed after colectomy in some of our patients with hyposplenism secondary to inflammatory bowel disease.

Colectomy for severe ulcerative colitis may be complicated by a syndrome including hypotension and DIC that suggests a gram negative septicaemia but is not always associated with positive blood cultures. ${ }^{1}$ This syndrome usually occurs in individuals whose colitis is complicated by hyposplenism and is similar to the generalised Schwartzman reaction. ${ }^{2}$ We have postulated that endotoxaemia may be responsible. ${ }^{3}$

However, the frequency of endotoxaemia and of bacteraemia in patients with ulcerative colitis is not established and there are no studies in which both have been assessed simultaneously in portal and systemic blood. We have therefore carried out such a study in a number of patients with ulcerative colitis undergoing colectomy.

\section{Methods}

\section{PATIENTS}

The indications for surgery were (1) chronic debilitating disease with frequent exacerbations requiring hospitalisation and intensive medical treatment, including corticosteroids (patients NH, PM, IK,

Received for publication 13 May 1980
IS and JB); (2) fulminating disease which failed to respond to conservative measures (patients $\mathrm{CK}$, CB and DT).

Six patients underwent panproctocolectomy with excision of the rectal stump and in two young males (DT and NT) the rectum was left in situ. On the basis of standard biochemical tests, all patients were considered to have normal liver function.

No patient was treated with antibiotics immediately before surgery but all received them in the immediate postoperative period. All patients received subcutaneous calcium heparin $(5000 \mu$ eight to 12 hourly) peri-operatively. There were no severe complications in the immediate postoperative period, although two patients developed local sepsis requiring drainage more than a week after colectomy and one underwent laparotomy for small bowel obstruction three weeks after operation.

The serum creatinine and haemotological parameters including haemogloblin, platelet count, and DIC screen (prothrombin time, thrombin time, serum fibrinogen, serum fibrin degradation products, kaolin cephalin coagulation time, and euglobulin lysis time) were measured repeatedly in the peri-operative period.

The splenic function of all the patients was 
assessed before surgery by the clearance of ${ }^{51} \mathrm{Cr}$ labelled heat damaged autologous red cells from the circulation $^{4}$ and by examination of the peripheral blood film.

At operation, $15 \mathrm{ml}$ samples of blood were taken as follows: (1) from a peripheral vein before the surgeon had opened the abdominal cavity; (2) from an accessible large mesenteric vein draining the colon, before any manipulation of the abdominal contents; (3) from peripheral and mesenteric veins during mobilisation of the colon; (4) from peripheral veins one and two days postoperatively.

Each sample was collected in a pyrogen-free syringe containing $0.5 \mathrm{ml}$ of $1: 1000$ heparin; iodine was used to clean the skin. Care was taken to expel any air drawn into the syringe during venepuncture. Ten millilitres of each sample was cultured for bacteria and the plasma from the remainder was drawn off and tested for the presence of endotoxin.

\section{BACTERIOLOGY}

Each sample was cultured aerobically and anaerobically in liquid media and pour plates.

\section{Liquid cultures}

(1) Three millilitres of blood was injected into a bottle containing $30 \mathrm{ml}$ of a Brain Heart Infusion Broth (Oxoid) and incubated in air at $37^{\circ} \mathrm{C}$. (2) Three millilitres of blood was injected into a modified BM broth with cooked meat particles. ${ }^{56}$ The bottles were steamed for 30 minutes and cooled to $37^{\circ} \mathrm{C}$ before use. These cultures were then incubated with the caps loose in an anaerobic jar. ${ }^{\text {? }}$

Both bottles were examined at 48 hours and one week and subcultured on to blood agar for aerobic incubation and BM-lysed blood agar $^{8}$ for anaerobic incubation.

\section{Solid cultures}

Pour plates were made by adding $1 \mathrm{ml}$ of the blood sample to $10 \mathrm{ml}$ of molten nutrient agar (Oxoid) at $50-55^{\circ} \mathrm{C}$ for aerobic incubation at $37^{\circ} \mathrm{C}$, and $1 \mathrm{ml}$ of blood was added to $10 \mathrm{ml}$ of molten BM agar at $50-55^{\circ} \mathrm{C}$ for anaerobic incubation. Anaerobic incubation was by the standard method of Collee et al. ${ }^{7}$; cultures were incubated at $37^{\circ} \mathrm{C}$ in an atmosphere of $90 \%$ hydrogen and $10 \% \mathrm{CO}_{2}$.

\section{ENDOTOXIN ASSAY}

This was by the limulus lysate test as described by Levin et al. ${ }^{9} 0.05 \mathrm{ml}$ of the chloroform extracted fraction were tested by incubation with $0.05 \mathrm{ml}$ of lysate; the remainder was stored at $-20^{\circ} \mathrm{C}$. Positive controls of normal serum to which standard endotoxin (Sigma Chemicals) had been added and nega- tive controls of normal serum were tested in parallel with the samples; the heparin used as anticoagulant was also tested with the lysate.

The samples were read after incubation overnight at $37^{\circ} \mathrm{C}$ and graded as negative $(-)$, positive $(+)$ if flocculation occurred, or strongly positive $(++)$ if solid gel developed.

Experiments with standard endotoxin showed that flocculation was associated with an endotoxin concentration of $0.0001 \mu \mathrm{g} / \mathrm{ml}$, gelation with a concentration of $0.001 \mu \mathrm{g} / \mathrm{ml}$.

Endotoxin testing of all glassware and heparin was consistently negative. All results were fully reproducible when the frozen serum was tested three to eight weeks later.

\section{Results}

The peri-operative course of the eight patients was uneventful and in no case was there evidence of DIC as shown by a negative DIC screen, normal platelet count, and maintenance of serum creatinine in each case. The first seven patients had normal splenic function, but patient JB had a prolonged half life for clearance of ${ }^{51} \mathrm{Cr}$-labelled heat damaged red cells, implying functional hyposplenism, despite having a normal blood film (28 min; normal 8.4 $18.5 \mathrm{~min})$. Blood cultures were positive in one individual (JK) though only during bowel mobilisation; the peripheral venous sample yielded a moderate growth of an alpha haemolytic streptococcus and the mesenteric blood grew Staphylococcus albus. In all other cases every sample was sterile.

Results of the endotoxin studies are shown in the table. Mesenteric endotoxaemia was present before mobilisation of the colon in three individuals and in another two patients during surgery. Endotoxin was present in peripheral blood in four patients during mobilisation; one of these had no endotoxin detectable in mesenteric blood. In only

Table Results of endotoxin assays on blood obtained at operation

\begin{tabular}{|c|c|c|c|c|}
\hline \multirow[t]{2}{*}{ Patient } & \multicolumn{2}{|c|}{ Before mobilisation } & \multicolumn{2}{|c|}{ During mobilisation } \\
\hline & $\begin{array}{l}\text { Peripheral } \\
\text { blood }\end{array}$ & $\begin{array}{l}\text { Portal } \\
\text { blood }\end{array}$ & $\begin{array}{l}\text { Peripheral } \\
\text { blood }\end{array}$ & $\begin{array}{l}\text { Portal } \\
\text { blood }\end{array}$ \\
\hline PM & - & - & + & ++ \\
\hline NH & - & - & - & + \\
\hline IK & - & - & - & - \\
\hline CK & - & - & - & - \\
\hline CB & - & - & - & - \\
\hline DT & - & + & + & ++ \\
\hline IS & - & + & + & - \\
\hline JB & + & + & - & $+t$ \\
\hline
\end{tabular}


the hyposplenic patient (JB) was there systemic endotoxaemia before bowel mobilisation. In each case limulus lysate testing of peripheral venous blood was negative on the first and second postoperative days.

\section{Discussion}

In contrast with the only previously published studies, ${ }^{1011}$ we were unable to demonstrate significant portal bacteraemia either before or during bowel mobilisation. We do not believe that the organisms cultured in IK originated from the bowel; almost certainly the staphylococcus was a contaminant. The bacteriological methods and media gave reliable recovery of demanding aerobic and anaerobic bacteria from very small inocula (5-10 variable organisms) in a laboratory model for blood culture studies $^{6}$ and it is very unlikely that our sensitive bacteriological technique would have failed to culture a significant organism, particularly $E$. coli or Bacteroides spp. Our findings are at variance with those of Eade and Brook, ${ }^{10}$ who found portal bacteraemia in 24 of 100 cases of ulcerative colitis submitted to surgery. These authors did not give details of their bacteriological technique but it is unlikely that technical differences would account for this discrepancy, although contamination during venesection could do so. Eade and Brook found the incidence of bacteraemia paralleled disease severity and it may be that their subjects had a more severe illness than ours, although three of our cases underwent emergency colectomy for fulminating disease.

It is possible that portal bacteraemia occurs intermittently in ulcerative colitis and the sterility of a total of 16 portal samples does not imply that the portal blood is always sterile; however, we feel that if there were a tendency for portal bacteraemia to occur it would have done so in some of our cases after the considerable colonic manipulation associated with surgery.

Most authors consider the limulus lysate test to be a sensitive test for endotoxin, ${ }^{9121315}$ though others express doubt about its validity. ${ }^{14-16}$ False positive results do occur in the presence of naturally occurring materials ${ }^{14}$ and normal serum contains an inhibitor which must be removed ${ }^{9}$; in this study it was removed by chloroform extraction. There is a poor relationship between the presence of bacteraemia and the results of the limulus lysate test ${ }^{16} 16$ which may imply a considerable false positive and negative rate. For this reason it was considered important to study both the endotoxin and bacteriological aspects together. The reported frequency of portal vein endotoxaemia assessed by the limulus lysate assay in non-colitis patients studied during surgery varies considerably from none of 16 patients $^{17}$ to 33 of $34,{ }^{18}$ although this high incidence reported by Jacob $^{18}$ has not been confirmed by other studies. ${ }^{17}{ }^{19-21}$ However, using radioimmunoassay, circulating endotoxins have been detected in the serum of ulcerative colitis patients ${ }^{22}$ who may also have lipopolysaccharide (LPS) antibodies. ${ }^{23}$ Systemic endotoxaemia may be experimentally produced by occluding the mesenteric arteries and portal vein endotoxaemia by occlusion of the portal vein. ${ }^{24}$

On the basis of this evidence, it has been suggested that ulcerative colitis is associated with increased portal vein absorption of endotoxin, related to mucosal damage, leading to generalised endotoxaemia. ${ }^{22}$ Our present studies and experimental work, however, suggest that this theory may be incorrect. Studies of the isolated gut sac of the rat show that endotoxin absorption obeys MichaelisMenton kinetics implying an active transport mechanism ${ }^{13}$ and it is possible that in ulcerative colitis this may be disrupted, leading to decreased passage of the molecule across the mucosa. We postulate that only in very severe mucosal damage, akin to experimentally induced mesenteric artery or portal vein occlusion, would endotoxin absorption occur down its concentration gradient and enter the portal system.

The chronic low titre, systemic endotoxaemia detectable by radioimmunoassay in ulcerative colitis is probably due to lymphatic absorption from the gut. The evidence for this is twofold; firstly, endotoxins are avidly removed by Kupffer cells, ${ }^{25}$ and secondly, experiments using Thiry-Vella segments have implied that most of the endotoxin entering the systemic circulation from the normal bowel does so through the bowel lymphatics. ${ }^{26}$ This would account for the finding in patient IS of systemic endotoxaemia in the absence of mesenteric vein endotoxin. The increased frequency and intensity of endotoxaemia occuring in some of our patients during mobilisation of the colon could lead to systemic complications, and it is interesting that endotoxin was detected in peripheral blood before operation only in the patient with diminished splenic function.

The relationship between endotoxin and reticuloendothelial function, in particular splenic function, is a complex one. The reticuloendothelial system is involved in the clearance and detoxification of endotoxin ${ }^{25}$ and also in the prevention of DIC by removal of microaggregates of fibrin. ${ }^{27}$ It has been suggested that the hyposplenism associated with coeliac disease is part of a generalised depression of reticuloendothelial function, ${ }^{28}$ but using the ${ }^{125} I$ macroaggregated albumin clearance test ${ }^{29}$ we have 
found no evidence of reticuloendothelial depression, other than that attributable to hyposplenism, in either coeliac disease or ulcerative colitis (unpublished observations). The observation in our hyposplenic patient does, however, indicate at least the possibility that there may be impaired removal of endotoxin in hyposplenic individuals with ulcerative colitis. What is in no doubt is our observation that during surgery temporary systemic endotoxaemia does occur. This did not precipitate disseminated intravascular coagulation in these patients possibly because of what is now our routine use of subcutaneous heparin before and after surgery. It could, however, have been responsible for the DIC which we have previously observed in similar patients. ${ }^{1}$

The authors would like to express their gratitude to $\mathrm{Dr} J$ Levin who gave us the limulus lysate, to Dr S Wilkinson for valuable technical advice, and to the surgeons of the Royal Hallamshire Hospital, Sheffield, for their patience in obtaining the mesenteric samples.

\section{References}

${ }^{1}$ Ryan FP, Smart RC, Holdsworth CD, Preston FE. Hyposplenism in inflammatory bowel disease. Gut 1978; 19: 50-5.

${ }^{2}$ Hjort PF, Rappaport SI. The Schwartzman reaction: pathogenetic mechanisms and clinical manifestations. Ann Rev Med 1965; 16: 135-68.

${ }^{3}$ Ryan FP, Timperley WR, Preston FE, Holdsworth CD. Cerebral involvement with disseminated intravascular coagulation in intestinal disease. J Clin Pathol 1977; 30: $551-5$.

${ }^{4}$ Marsh GW, Lewis SM, Szur L. The use of ${ }^{51} \mathrm{Cr}$-labelled heat-damaged red cells to study splenic function. Br J Haematol 1966; 12: 161-6.

${ }^{5}$ Deacon AG, Duerden BI, Holbrook WP. Gas-liquid chromatographic analysis of metabolic products in the identification of bacteroidaceae of clinical interest. J Med Microbiol 1978; 11 : 81-99.

${ }^{6}$ Collee JG, Duerden BI, Brown R. Recovery of anaerobic bacteria from small inocula: a model for blood culture studies. J Clin Pathol 1977; 30: 609-14.

${ }^{7}$ Collee JG, Watt B, Fowler EB, Brown R. An evaluation of the Gaspak system in the culture of anaerobic bacteria. J Appl Bacteriol 1972; 35: 71-82.

${ }^{8}$ Holbrook WP, Ogston SA, Ross PW. A method for the isolation of Bacteroides melaninogenicus from the human mouth. J Med Microbiol 1978; 11: 203-7.

${ }^{9}$ Levin J, Tomasulo PA, Oser RS. Detection of endotoxin in human blood and demonstration of an inhibitor. J Lab Clin Med 1970; 75: 903-11.

${ }^{10}$ Eade MN, Brook BN. Portal bacteraemia in cases of ulcerative colitis submitted to colectomy. Lancet $1969 ; 1: 1008-9$.

${ }^{11}$ Brooke BN, Slaney G. Portal bacteraemia in ulcerative colitis (letter). Lancet 1958; 1 : 1206-7.
${ }^{12}$ Cooper JF, Levin J, Wagner HN Jr. Quantitative comparison of in-vitro and in-vivo methods for the detection of endotoxin. J Lab Clin Med 1971; 78: 138-48.

${ }^{13}$ Nolan JP, Hare DK, McDevitt JJ, Ali MV. In vitro studies of intestinal endotoxin absorption. I. Kinetics of absorption in the isolated everted gut sac. Gastroenterology 1977; 72: 434-9.

${ }^{14}$ Elin RJ, Wolff SM. Nonspecificity of the limbus amebocyte lysate test: positive reactions with polynucleotides and proteins. J Infect Dis 1973; 128: 349-52.

${ }^{15}$ Stumacher RJ, Kovnatt MJ, McCabe WR. Limitations of the usefulness of the limulus assay for endotoxin. N Engl J Med 1973; 288: 1261-4.

${ }^{16}$ Feldman S, Pearson TA. The limulus test and gramnegative bacillary sepsis. Am J Dis Child 1974; 128: 172-4.

${ }^{17}$ Bailey ME. Endotoxin, bile salts and renal function in obstructive jaundice. Br J Surg 1976; 63: 774-8.

${ }^{18} \mathrm{Jacob}$ AI, Goldberg PK, Bloom N, Degenshein GA, Kozinn PJ. Endotoxin and bacteria in portal blood. Gastroenterology 1977; 72: 1268-70.

${ }^{19}$ Ansell J, Widrich W, Johnson W, Fine J. Endotoxin and bacteria in portal blood (letter). Gastroenterology 1977; $73: 1190$.

${ }^{20}$ Triger DR, Boyer TD, Levin J. Portal and systemic bacteraemia and endotoxaemia in liver disease. Gut 1978; 19: 935-9.

${ }^{21}$ Prytz H, Holst-Christensen J, Korner B, Liehr $\mathbf{H}$. Portal venous and systemic endotoxaemia in patients without liver disease and endotoxaemia in patients with cirrhosis. Scand J Gastroenterol 1976; 11 : 857-63.

${ }^{22}$ Aoki K. A study of endotoxaemia in ulcerative colitis and Crohn's disease: I. Clinical study. Acta Med Okayama 1978; 32(2): 147-58.

${ }^{23}$ Tai C. Clinical and experimental endotoxaemia related to the intestinal ischaemia detection of endotoxin by means of radioimmunoassay. Okayama Med J 1974; 28: $111-4$.

${ }^{24}$ Olcay I, Kitahama A, Miller RH, Drapanas T, Trejo RA, Di-Luzio NR. Reticuloendothelial dysfunction and endotoxemia following portal vein occlusion. Surgery 1974; 75: 64-70.

${ }^{25}$ Carey FJ, Braude AI, Zalesky M. Studies with radioactive endotoxin. III. The effect of tolerance on the distribution of radioactivity after intravenous injection of Escherichia coli endotoxin labelled with $\mathrm{Cr}^{51}$. J Clin Invest 1958; 37: 441-57.

${ }^{26} \mathrm{Gans} \mathrm{H}$, Matsumuto $\mathrm{K}$. The escape of endotoxin from the intestine. Surg Gynecol Obstet 1974; 139: 395-402.

${ }^{27}$ Lee L. Reticuloendothelial clearance of circulating fibrin in the pathogenesis of the generalised Schwartzman reaction. J Exp Med 1962; 115: 1065-82.

${ }^{28}$ McCarthy CF, Fraser ID, Evans KT, Read AE. Lymphoreticular dysfunction in idiopathic steatorrhoea. Gut 1966; 7: 140-8.

${ }^{29}$ Iio M, Wagner HN Jr. Studies of the reticuloendothelial system (R.E.S.). I: Measurement of the phagocytic capacity of the R.E.S. in man and the dog. $J$ Clin Invest 1963 ; 42: 417-26. 